9th A. Friedmann International Seminar and

3rd Casimir Symposium 2015

International Journal of Modern Physics: Conference Series

Vol. 41 (2016) 1660127 (10 pages)

(C) The Author(s)

DOI: $10.1142 /$ S2010194516601277

\title{
Cosmological model favored by the holographic principle
}

\author{
Irina Dymnikova \\ A. F. Ioffe Physico-Technical Institute, Politekhnicheskaja 26, \\ St. Petersburg, 194021 Russia \\ University of Warmia and Mazury, Stoneczna 54, 10-710 Olsztyn, Poland \\ irina@matman.uwm.edu.pl \\ Anna Dobosz* and Bożena Sołtysek ${ }^{\dagger}$ \\ University of Warmia and Mazury, Stoneczna 54, 10-710 Olsztyn, Poland \\ *dobosz@uwm.edu.pl; †bozena@matman.uwm.edu.pl \\ Received 28 September 2015 \\ Published 18 March 2016
}

\begin{abstract}
We present a regular spherically symmetric cosmological model of the Lemaitre class distinguished by the holographic principle as the thermodynamically stable end-point of quantum evaporation of the cosmological horizon. A source term in the Einstein equations connects smoothly two de Sitter vacua with different values of cosmological constant and corresponds to anisotropic vacuum dark fluid defined by symmetry of its stress-energy tensor which is invariant under the radial boosts. Global structure of spacetime is the same as for the de Sitter space-time. Cosmological evolution goes from a big initial value of the cosmological constant towards its presently observed value.
\end{abstract}

Keywords: Dark energy; cosmological constant; holographic principle.

PACS numbers: 98.80; 98.80.-k; 95.36.+x

\section{Introduction}

Astronomical observations testify convincingly that our Universe is dominated in above $73 \%$ of its density by a dark energy with a negative pressure $p=w \rho$ with $w<-1 / 3$, responsible for its accelerated expansion. Existence of a dark energy is known since late $90-$ s. $^{1-3}$ The best fit for a dark energy $w=-1$ (see Ref. 4 and references therein) corresponds to the Einstein cosmological term $\lambda g_{\mu \nu}$; the cosmological constant $\lambda$ is related to the de Sitter vacuum density $\rho_{v a c}$ by $\lambda=8 \pi G \rho_{v a c}$. The Einstein cosmological term is plagued by the problem of cosmological constant:

This is an Open Access article published by World Scientific Publishing Company. It is distributed under the terms of the Creative Commons Attribution 4.0 (CC-BY) License. Further distribution of this work is permitted, provided the original work is properly cited. 
The inflationary paradigm needs a large value $\Lambda=8 \pi G \rho_{v a c}$ at the beginning, the quantum field theory estimates $\rho_{v a c}$ by $\rho_{P l}$ which exceeds the observational value by 123 orders of magnitude, and the Einstein equations require $\rho_{v a c}=$ const.

Various models developed to describe dynamical dark energy, typically assume $\rho_{v a c}=0$ and introduce a dark energy of non-vacuum origin which mimics cosmological constant when necessary (for a review see Refs. 5 and 6).

Our approach is based on the model-independent description of vacuum dark energy suggested by the Petrov classification of stress-energy tensors and related to the space-time symmetry. ${ }^{7,8}$ The Einstein cosmological term, associated with a vacuum stress-energy tensor of maximal symmetry $T_{\mu \nu}=\rho_{v a c} g_{\mu \nu}$, provides accelerated expansion of the Universe independently on a particular model for $\rho_{v a c} .{ }^{9}$ Its maximal symmetry can be reduced to the case when less than three space-like eigenvalues are equal to the time-like eigenvalue. ${ }^{7,10}$ This leads to a dynamical vacuum dark energy represented by anisotropic vacuum dark fluid which can both be distributed and form compact objects. ${ }^{11}$ It generates regular space-times with the de Sitter interior, asymptotically de Sitter at infinity with $\lambda<\Lambda$, whose existence follows from requirements of regularity and of the validity of the weak energy conditions for a source term in the Einstein equations. ${ }^{8}$

Time-evolving and space-inhomogeneous cosmological term ${ }^{7}$ generates regular cosmological models dominated by dynamical vacuum dark energy (see Ref. 12 and references therein). In this talk we present the model distinguished by the holographic principle ${ }^{13}$ in which cosmological evolution is dictated by quantum dynamics of the cosmological horizon and goes from initial a GUT scale value towards presently observed value without a special fine-tuning.

\section{Regular Spherically Symmetric Space-Time}

The Einstein equations admit the class of regular spherically symmetric solutions with a source term ${ }^{10-13}$

$$
T_{t}^{t}=T_{r}^{r} ; \quad T_{\theta}^{\theta}=T_{\phi}^{\phi}
$$

asymptotically de Sitter in the origin and at the late times

$$
\Lambda \delta_{\mu}^{\nu} \underset{r \rightarrow 0}{\longleftarrow} T_{\mu}^{\nu} \underset{r \rightarrow \infty}{\longrightarrow} \lambda \delta_{\mu}^{\nu}
$$

Equation of state, following from $T_{\mu ; \nu}^{\nu}=0$, reads

$$
p_{\perp}=-\rho-\frac{r}{2} \rho^{\prime}
$$

where $\rho(r)=T_{t}^{t}$ is the energy density, $p_{r}(r)=-T_{r}^{r}$ is the radial pressure, and $p_{\perp}(r)=-T_{\theta}^{\theta}=-T_{\phi}^{\phi}$ is the tangential pressure for anisotropic perfect fluid.

A metric generated by a source term specified by (1), belongs to the class of metrics

$$
d s^{2}=g(r) d t^{2}-\frac{d r^{2}}{g(r)}-r^{2} d \Omega^{2}
$$


A density component of $T_{\mu}^{\nu}$ can be written as ${ }^{14}$

$$
T_{t}^{t}(r)=\rho(r)+(8 \pi G)^{-1} \lambda ; \quad \rho(r) \rightarrow(8 \pi G)^{-1} \Lambda \text { as } r \rightarrow 0 .
$$

Geometry is described by the metric function ${ }^{14}$

$$
g(r)=1-\frac{2 G \mathcal{M}(r)}{r}-\frac{\lambda r^{2}}{3},
$$

whose asymptotes are the de Sitter metrics with $\lambda$ as $r \rightarrow \infty$ and $(\Lambda+\lambda)$ as $r \rightarrow 0$. The mass function $\mathcal{M}(r)$ is given by

$$
\mathcal{M}(r)=4 \pi \int_{0}^{r} \rho(x) x^{2} d x .
$$

Geometry defined by (6) has three characteristic length scales:

$$
r_{g}=2 G M ; \quad r_{0}=\sqrt{\frac{3}{\Lambda}} ; l=\sqrt{\frac{3}{\lambda}},
$$

where $M=4 \pi \int_{0}^{\infty} \rho(r) r^{2} d r$ is the mass parameter, characteristic length scale $r_{0}$ is related to the de Sitter vacuum in the origin $\rho_{0}=(8 \pi G)^{-1} \Lambda$ and $l$ to the background vacuum density $\rho_{\lambda}=(8 \pi G)^{-1} \lambda$.

The behavior of the metric function $g(r)$ is dictated by the number of vacuum scales through behavior of the transversal pressure $p_{\perp}$ in a source term. ${ }^{12}$ In the case of two vacuum scales there are at most three horizons. ${ }^{15}$

In the 3-horizon case, the geometry describes a regular black hole with de Sitter center in the asymptotically de Sitter space, ${ }^{14}$ shown in Fig. 1, or the Lemaitre class cosmological model with the second phase transition at the QCD scale. ${ }^{12}$

For numerical calculations we adopt the density profile ${ }^{10}$

$$
\rho(r)=\rho_{0} \exp \left(-\frac{r^{3}}{r_{0}^{2} r_{g}}\right),
$$

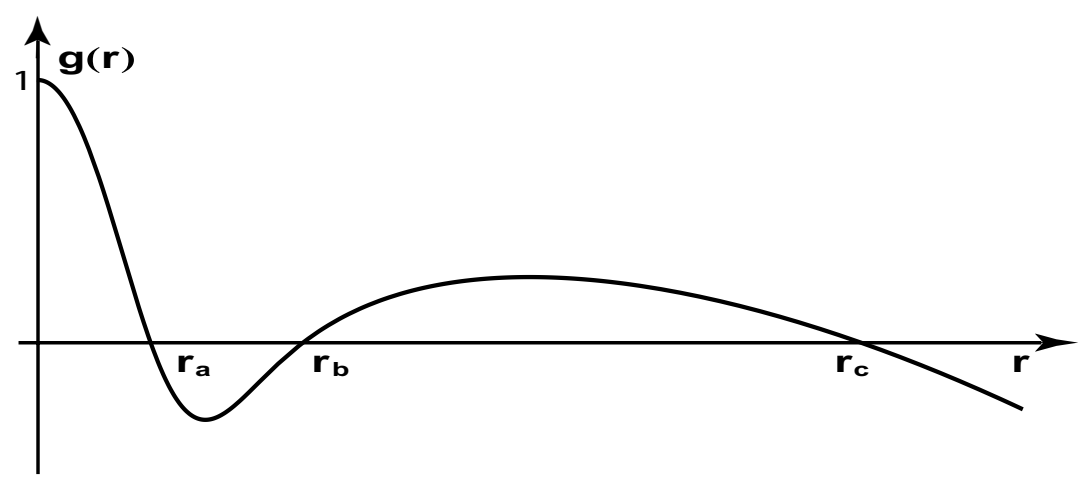

Fig. 1. Typical behavior of $g(r)$ for the case of 3 horizons: a black (white) hole horizon $r_{b}$, a cosmological horizon $r_{c}$, an internal Cauchy horizon $r_{a}$ related to the de Sitter center. 


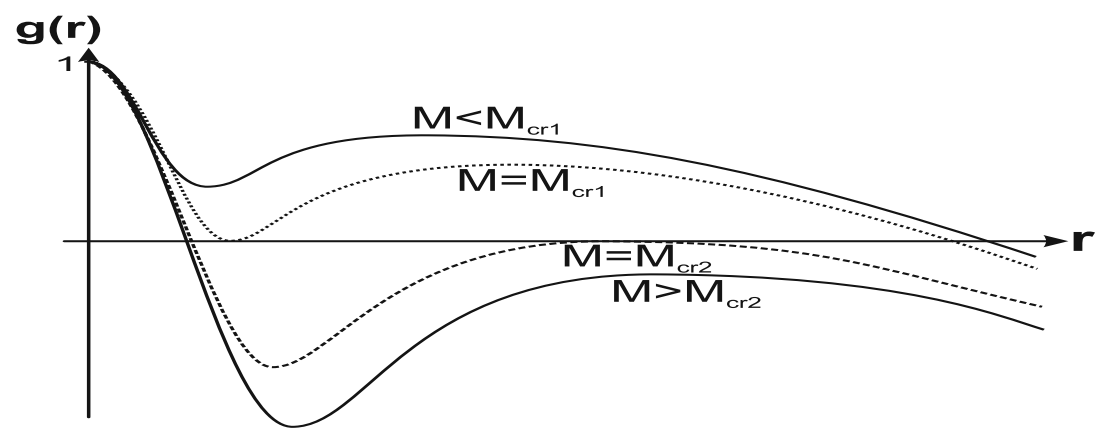

Fig. 2. Metric function for double-horizon and one-horizon space-times.

which describes vacuum polarization effects leading to de Sitter interior in the simple semi-classical model for vacuum polarization in the gravitational field. ${ }^{8,16}$

For a 3-horizon space-time the mass parameter is confined within $M_{c r 1}<M<$ $M_{c r 2}$. Values $M_{c r 1}$ and $M_{c r 2}$ depend on the characteristic parameter $q$ relating two vacuum scales, $\Lambda$ and $\lambda: q=\sqrt{\frac{\lambda}{\lambda}}$. Four other possible configurations are shown in Fig. 2. Double-horizon configurations $M_{c r 1}$ and $M_{c r 2}$ represent, respectively, an extreme regular black hole and the regular modification of the Nariai solution. Configurations $M<M_{c r 1}$ and $M>M_{c r 2}$ represent 1-horizon space-times with the global structure of the de Sitter space-time.

For 1-horizon space-times (see Fig. 3) the global structure of space-time is the same as for de Sitter geometry, but vacuum dark energy is time-evolving an spaceinhomogeneous. Static observer exist in the $R$ regions, where $g(r)>0$. $T$ regions, where $g(r)<0$, correspond to regular cosmological models of the Kantowski-Sachs type with the null bang from the horizon.. ${ }^{15}$ The Lemaitre observers have for their disposal the whole manifold. In the Lemaitre cosmological models evolution starts with a nonsingular non-simultaneous bang from a regular time-like surface, is followed by an anisotropic stage and goes to the de Sitter stage at late times. ${ }^{15,17}$
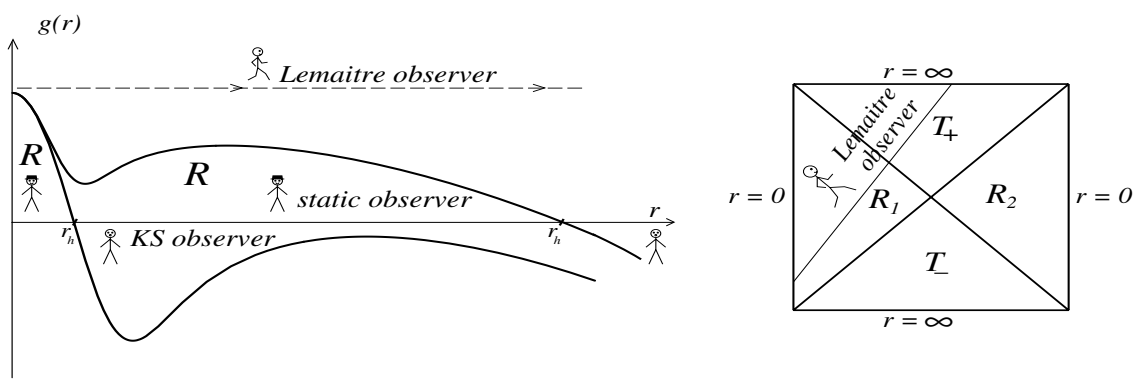

Fig. 3. Spherically symmetric 1-horizon space-time. 


\section{Holographic Principle and Triple-Horizon Space-Time}

The holographic principle was formulated ${ }^{18}$ as the requirement that the number of independent quantum degrees of freedom contained in a given spatial volume is bounded from above by the surface area of the region. It led to a conjecture that a physical system can be completely specified by data stored on its boundary. ${ }^{19,20}$

Quantum dynamics of the cosmological horizon distinguishes one of 1-horizon space-times as the only thermodynamically stable final product of its evaporation. ${ }^{13}$

The Gibbons-Hawking temperature on a horizon $r_{h}$ is given by ${ }^{21}$

$$
k T_{H}=\frac{\hbar c}{4 \pi}\left|g^{\prime}\left(r_{h}\right)\right|
$$

The entropy and thermodynamical energy (in the units $c=G=\hbar=1$ ) $\operatorname{are}^{22}$

$$
S=\pi r_{h}^{2} ; \quad E=\frac{\left|g^{\prime}\right|}{g}\left(\frac{A_{h}}{16 \pi}\right)^{\frac{1}{2}}=\frac{\left|g^{\prime}\right|}{g} \frac{r_{h}}{2},
$$

where $A_{h}$ is the horizon area.

A specific heat $C_{h}=\frac{d E_{h}}{d T_{h}}$ is given by ${ }^{23}$

$$
C_{h}=\frac{2 \pi r_{h}}{g^{\prime}\left(r_{h}\right)+g^{\prime \prime}\left(r_{h}\right) r_{h}} .
$$

The cosmological horizon $r_{h}$ of an observer in the $R$-region $0 \leq r<r_{h}$ is the future boundary of his manifold, the 2 -nd law of thermodynamics, $d S_{h} \geq 0$, requires $d r_{h} \geq 0$, and the horizon moves outwards ( ${ }^{24}$ and references therein). Increasing of a cosmological horizon leads to decreasing of the mass parameter $M .^{23-25}$

1-horizon space-time with $M>M_{c r 2}$ and three extrema shown in Fig. 2, evolves during evaporation towards the double-horizon space-time with $M=M_{c r 2}$, with zero temperature by virtue of (10). It is thermodynamically unstable since in a maximum $g^{\prime \prime}<0$ and hence $C_{h}<0$.

Now let us consider a 1-horizon space-time with the metric function shown in Fig. 4, which does not have extrema in the $T$-region $r>r_{h}$ but an inflection point distinguished by two conditions: $g^{\prime}\left(r_{i}\right)=0 ; g^{\prime \prime}\left(r_{i}\right)=0$.

Quantum evaporation of the cosmological horizon in this case goes towards the point where decreasing mass achieves a certain critical value $M=M_{c r}$ at which the metric function vanishes. This is the point where two critical masses $M_{c r 1}$ i $M_{c r 2}$ coincide at a certain value $q=q_{c r}$, which corresponds to the triple horizon $r_{h}=r_{t}$, where the metric function, and its first and second derivatives vanish.

Evolution starts from $M>M_{c r}$ (the lower curve in Fig. 4). Cosmological horizon moves outwards, temperature goes to zero, and vacuum density decreases unless it achieves a finite value corresponding to the triple-horizon state. The specific heat tends to infinity at the triple horizon which testifies that the triple-horizon space-time is thermodynamically stable final product of quantum evaporation of the cosmological horizon in the course of evolution of a 1-horizon space-time with an inflection point. ${ }^{13}$ 


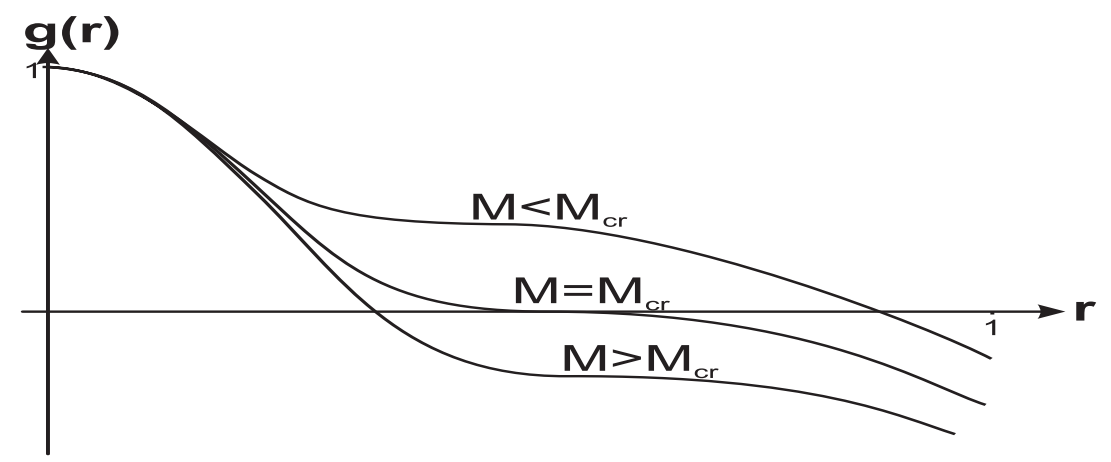

Fig. 4. Metric function for 1-horizon case with the inflection point.

Evaporation stops completely $\left(T_{h}=0 ; \quad C_{h} \rightarrow \infty\right)$ at the finite non-zero value of the vacuum density. The value of the cosmological constant is tightly fixed by the final point in the evaporation of the cosmological horizon. The triple horizon satisfies three algebraic equations:

$$
g\left(r_{t}\right)=0 ; \quad g^{\prime}\left(r_{t}\right)=0 ; \quad g^{\prime \prime}\left(r_{t}\right)=0,
$$

which define uniquely $M_{c r}, r_{t}$, and $q_{c r}$.

Choosing the density profile (9) and adopting $\rho_{0}=\rho_{G U T}$ with $E_{G U T}=$ $10^{15} \mathrm{GeV}$ (then $\rho_{G U T}=5 \times 10^{77} \mathrm{~g} / \mathrm{cm}^{3}$ and $r_{0}=2.4 \times 10^{-25} \mathrm{~cm}$ ), we find ${ }^{13}$

$$
M_{c r}=2.33 \times 10^{56} \mathrm{~g} ; \quad q_{c r}^{2}=1.37 \times 10^{107} ; r_{t r}=5.4 \times 10^{28} \mathrm{~cm} .
$$

This gives the remarkable coincidence of the calculated parameter $q_{c r}^{2}$ with that corresponding to our universe with ${ }^{4,5}$

$$
\rho_{\lambda(o b s)} \simeq 3.6 \times 10^{-30} \mathrm{~g} / \mathrm{cm}^{3} \Longrightarrow q^{2}=\rho_{G U T} / \rho_{\lambda(o b s)}=1.39 \times 10^{107} .
$$

The de Sitter horizon is $l=9 \times 10^{28} \mathrm{~cm}$.

\section{The Lemaitre Cosmological Model with Vacuum Dark Fluid}

We introduce an expanding coordinates related to a certain radial time-like geodesic family. For the metric (4) time-like radial geodesic satisfies the equations

$$
\left(\frac{d r}{d \tau}\right)^{2}=E^{2}-g(r) ; \quad \frac{d t}{d \tau}=\frac{E}{g(r)},
$$

where $E$ is the constant of motion along a geodesic.

Relating the reference frame to geodesics with a fixed value of $E$, we perform a transition from the coordinates $(r, t)$ to the coordinates $(R, \tau)$ with transformation:

$$
\begin{aligned}
\partial_{\tau} r & = \pm \sqrt{E^{2}-g(r)} ; & \partial_{R} r & =\sqrt{E^{2}-g(r)} \\
\partial_{\tau} t & =\frac{E}{g(r)} ; & \partial_{R} t & = \pm \frac{E^{2}-g(r)}{g(r) E},
\end{aligned}
$$


where $\tau$ is the proper time along a geodesic, $R$ is the new radial coordinate (the congruence parameter of geodesics family). The plus and minus signs refer to growing and falling $r(\tau)$ (expanding and contracting models), respectively.

The resulting metric has the form

$$
d s^{2}=d \tau^{2}-\frac{E^{2}-g(r(R, \tau))}{E^{2}} d R^{2}-r^{2}(R, \tau) d \Omega^{2} .
$$

For expanding models $\partial_{\tau} r=\partial_{R} r$, and $r$ is a function of $R+\tau$. This metric represents a special case of the Lemaitre class of metrics.

A Lemaitre class models with anisotropic fluid are described by the line element

$$
d s^{2}=d \tau^{2}-e^{2 \nu(R, \tau)} d R^{2}-r^{2}(R, \tau) d \Omega^{2} .
$$

Coordinates $R, \tau$ are the Lagrange comoving coordinates.

Generic behavior of the Lemaitre class models is related to the function $g(r)$ expressed by (6) in terms of the mass function (7).

The behavior of pressures at early times is shown in Fig. 5 for the density profile (9). For the metric (19), the Einstein equations with the SET (1) $\operatorname{read}^{26}$

$$
\begin{gathered}
8 \pi G p_{r}=\frac{1}{r^{2}}\left(e^{-2 \nu} r^{2}-2 r \ddot{r}-\dot{r}^{2}-1\right), \\
8 \pi G p_{\perp}=\frac{e^{-2 \nu}}{r}\left(r^{\prime \prime}-r^{\prime} \nu^{\prime}\right)-\frac{\dot{r} \dot{\nu}}{r}-\ddot{\nu}-\dot{\nu}^{2}-\frac{\ddot{r}}{r}, \\
8 \pi G \rho=-\frac{e^{-2 \nu}}{r^{2}}\left(2 r r^{\prime \prime}+r^{2}-2 r r^{\prime} \nu^{\prime \prime}\right)+\frac{1}{r^{2}}\left(2 r \dot{r} \dot{\nu}+\dot{r}^{2}+1\right), \\
8 \pi G T_{t}^{r}=\frac{2 e^{-2 \nu}}{r}\left(\dot{r}^{\prime}-r^{\prime} \dot{\nu}\right),
\end{gathered}
$$

where dots and primes stand for $\partial_{\tau}$ and $\partial_{R}$.
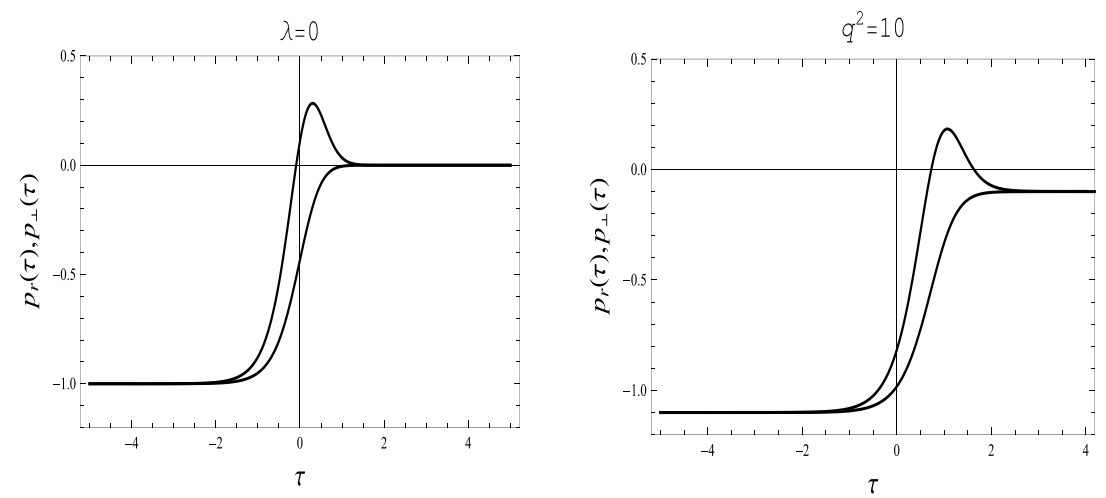

Fig. 5. Radial and tangential pressures for $\lambda=0$ and for $q^{2}=10$. 
In the comoving reference frame the component $T_{t}^{r}$ vanishes and the equation (23) is integrated giving ${ }^{27}$

$$
e^{2 \nu}=\frac{r^{\prime 2}}{1+f(R)},
$$

where $f(R)$ is an arbitrary function.

Putting (24) into (20), we obtain the equation of motion

$$
\dot{r}^{2}+2 r \ddot{r}+8 \pi G p_{r} r^{2}=f(R) .
$$

Taking into account that $p_{r}+\rho=0$, the first integration of (25) gives

$$
\dot{r}^{2}=\frac{2 G \mathcal{M}(r)}{r}+f(R)+\frac{F(R)}{r} .
$$

An arbitrary integration constant $F(R)$ should be chosen equal to zero for models regular at $r=0$ since $\mathcal{M}(r) \rightarrow 0$ as $r \rightarrow 0$ where $\rho(r) \rightarrow \rho_{0}<\infty$.

Second integration of (25) gives

$$
\tau-\tau_{0}(R)=\int \frac{d r}{\sqrt{2 G \mathcal{M}(r) / r+f(R)}},
$$

where $\tau_{0}(R)$ is an arbitrary function called the bang-time function. ${ }^{28}$

In the regular case asymptotically de Sitter in the $R$-region near $r=0$ evolution starts from the time-like regular surface $r(R, \tau)$, and the solution near this surface reduces to

$$
\tau-\tau_{0}(R)=\int \frac{d r}{\sqrt{r^{2} / r_{0}^{2}+f(R)}} .
$$

For $f(R)=0$ equation (28) gives at small $r$ the expansion law

$$
r=r_{0} e^{\left(\tau-\tau_{0}(r)\right) / r_{0}} ; \quad e^{2 \nu}=\frac{r^{2}}{r_{0}^{2}}\left[\frac{d \tau_{0}(R)}{d R}\right]^{2}
$$

and the metric takes the FRW form with the de Sitter scale factor

$$
d s^{2}=d \tau^{2}-r_{0}^{2} e^{2 c \tau / r_{0}}\left(d q^{2}+q^{2} d \Omega^{2}\right),
$$

where the variable $q=e^{\tau_{0}(R) / r_{0}}$ is introduced to transform the metric to the FRW form. It describe a nonsingular nonsimultaneous de Sitter bang from the surface $r\left(\tau-\tau_{0}(R) \rightarrow-\infty\right)=0 .{ }^{15,17}$

For the spatially flat model $(f(R)=0)$, the line element $(19)$ takes the form

$$
d s^{2}=d \tau^{2}-b^{2}(\tau, R) R^{2}-r^{2}(\tau, R) d \Omega^{2},
$$

where we have introduced two scale factors: $r(\tau, R)$ and $b(\tau, R) \equiv r^{\prime}(\tau, R)$.

We choose an arbitrary integration "constant" $\tau_{0}(R)=-R$. In this case $\dot{r}(\tau+$ $R)=d r / d(\tau+R)$. Numerical integration of the equation of motion in the Lemaitre coordinates shows an exponential growth of both scale factors at the beginning when $p_{\perp} \simeq p_{r}=-\rho$, followed by an anisotropic Kasner-like stage when the anisotropy of the pressures leads to an anisotropic expansion. The behavior of two scale factors is shown in Fig. 6, 7. At late times the metric (19) takes the FRW form with the de Sitter scale factor for $\rho_{v a c}=\rho_{\lambda}$. 

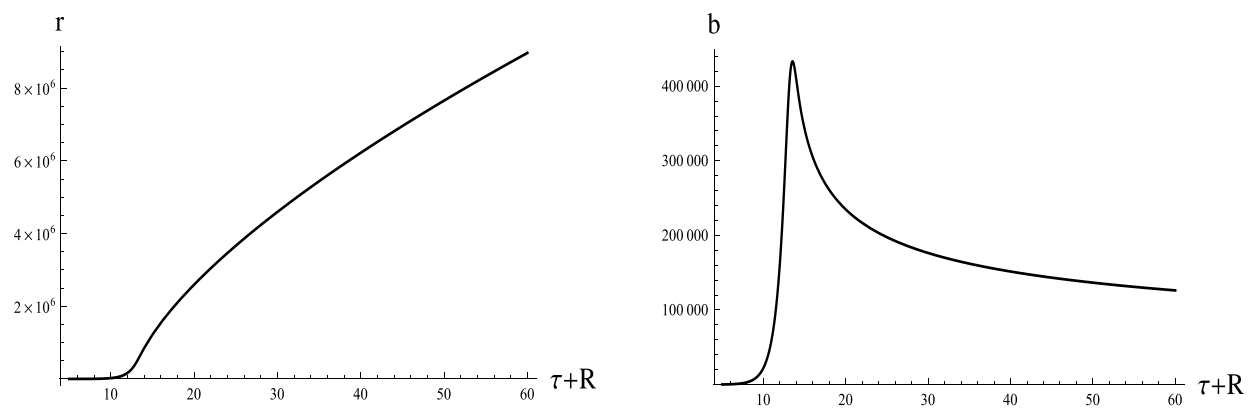

Fig. 6. Behavior of the scale factors $r(\tau+R)$ and $b(\tau+R)$.
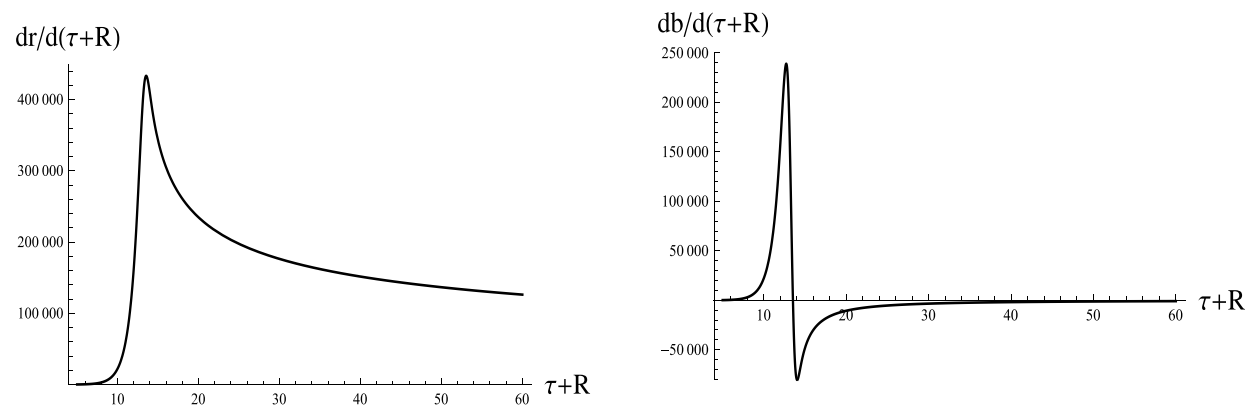

Fig. 7. Behavior of the velocities $\dot{r}(\tau+R)$ and $\dot{b}(\tau+R)$.

\section{Summary}

- Cosmological model preferred by the holographic principle, describes evolution of the universe from the early inflation to the present stage with the accelerated expansion due to relaxing dynamical vacuum dark energy.

- Cosmological evolution starts at the GUT scale and finishes at the triple horizon $r_{t r} \simeq 5.14 \times 10^{28} \mathrm{~cm}$, which is absolutely thermodynamically stable state with zero quantum temperature of the cosmological horizon.

- The triple-horizon space-time is distinguished by the quantum dynamics of the horizon as the only thermodynamically stable final product of its evaporation.

- The basic generic features of the triple horizon $r_{h}=r_{t r}$ are: ${ }^{13}$

(a) the finite entropy,

(b) zero temperature,

(c) infinite positive specific heat,

(d) zero curvature and zero transversal pressure.

The parameter $q^{2}=\rho_{G U T} / \rho_{\lambda(o b s)}=1.37 \times 10^{107}$ is found to be very close to the value $q^{2}=1.39 \times 10^{107}$ corresponding to the observable dark energy density ${ }^{5}$ $\rho_{\lambda(\mathrm{obs})} \simeq 3.6 \times 10^{-30} \mathrm{~g} \mathrm{~cm}^{-3}$. 


\section{References}

1. A. G. Riess et al., Astron. J. 116, 1009 (1998); A. G. Riess et al., Astron. J. 117, 707 (1999).

2. S. J. Perlmutter et al., Astrophys. J. 517, 565 (1999).

3. N. A. Bahcall et al., Science 284, 1481 (1999).

4. V. Springel, C. S. Frenk and S. M. D. White, Nature 440, 1137 (2006).

5. E. J. Copeland, in Invisible Universe, AIP Conf. Proc., Vol. 1241 (2010), p. 125.

6. V. Sahni, A. Shafiello and A. Starobinsky, Astrophys. J. 793, L40 (2014).

7. I. G. Dymnikova, Phys. Lett. B 472, 33 (2000).

8. I. Dymnikova, Class. Quant. Grav. 19, 725 (2002).

9. E. B. Gliner and I. G. Dymnikova, Sov. Astron. Lett. 1, 93 (1975).

10. I. Dymnikova, Gen. Rel. Grav. 24, 235 (1992).

11. I. Dymnikova and E. Galaktionov, Phys. Lett. B 645, 358 (2007).

12. K. Bronnikov, I. Dymnikova and E. Galaktionov, Class. Quant. Grav. 29, 095025 (2012).

13. I. Dymnikova, Int. J. Mod. Phys. D 21, 124007 (2012).

14. I. Dymnikova and B. Soltysek, Gen. Rel. Grav. 30, 1775 (1998).

15. K. A. Bronnikov, A. Dobosz and I. Dymnikova, Class. Quant. Grav. 20, 3797 (2003).

16. I. Dymnikova, Int. J. Mod. Phys. D 5, 529 (1996).

17. I. Dymnikova, A. Dobosz, M. Filchenkov and A. Gromov, Phys. Lett. B 506, 351 (2001); I. Dymnikova, A. Dobosz, M. Filchenkov and A. Gromov, Grav. Cosmol. 6 Suppl., 78 (2000).

18. G. 't Hooft, Dimensional reduction in quantum gravity, arXiv:9310026.

19. L. Susskind, J. Math. Phys. 36, 6377 (1995).

20. R. Bousso, Class. Quant. Grav. 17, 997 (2000).

21. G. W. Gibbons and S. W. Hawking, Phys. Rev. D 15, 2738 (1977).

22. T. Padmanabhan, Class. Quant. Grav. 19, 5387 (2002).

23. I. Dymnikova and M. Korpusik, Phys. Lett. B 685, 12 (2010).

24. I. Dymnikova and M. Korpusik, Entropy 13, 1967 (2011).

25. A. Gomberoff and C. Teitelboim, Phys. Rev. D 67, 104024 (2003).

26. L. D. Landau and E. M. Lifshitz, Classical Theory of Fields (Pergamon Press, Oxford, 1975).

27. R. C. Tolman, Proc. Nat. Acad. Sci. USA 20, 169 (1934).

28. D. W. Olson and J. Silk, Astrophys. J. 233, 395 (1979). 